\title{
Low-uncertainty absolute radiometric calibration of a $\mathrm{CCD}$
}

\author{
Alejandro Ferrero, Joaquin Cam- \\ pos and Alicia Pons
}

\begin{abstract}
.
The use of CCD detectors for radiometric measurements has proven to be very interesting for a variety of fields. This work presents an experimental method for the low-uncertainty calibration of the spectral radiant exposure responsivity of a CCD detector. It contains the description of the experimental setup as well as the analysis of the various sources of uncertainty. The overall result shows that the calibration procedure-related uncertainty is only $0.18 \%$ as long as the temperature is kept constant and the field of view is restricted to angles under 13 .
\end{abstract}

\section{Introduction}

¿From the moment CCD detectors were invented in 1969 by Willard S. Boyle and George E. Smith at Bell Telephone Laboratories [1], they have aroused a growing interest among the scientific community [2]. Thanks to their higher linearity features, to their increased sensitivity within the visible range and to the higher accessibility to the experimental data that they offer, CCD detectors soon superseded photographic plates and revolutionized astronomic instrumentation, which was key to its rapid development and evolution.

Nowadays CCDs are a key component in many scientific and technological fields used as highlyefficient instruments to characterize complex scenes. In this sense, CCD detectors are employed, for instance, in a wide variety of industrial applications, for color measurements [3, 4, 5], Astrofsica, iluminacin $[6,7]$, in Astrophysics, in illumination $[6,7]$ (characterization of the spatial distribution of radiant sources) or even in artificial vision[8]. Their increased popularity is due mainly to them showing high resolution, high quantum efficiency, broad spectral response, low noise, linearity, geometric fidelity, fast response, reduced dimensions, low consumption and long life. Moreover, since the individual detectors that make up the array have all the same dimensions

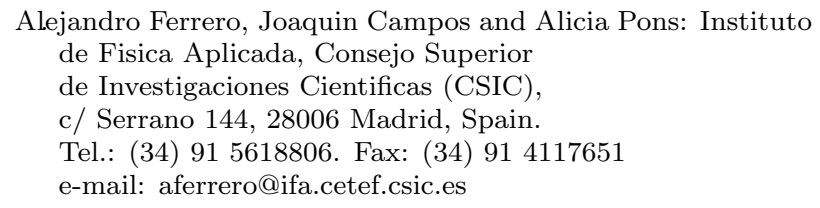

and geometry, the CCD offers a reliable spatial quantization, which allows a precise representation of the information on a standard monitor[9].

In Radiometry, CCDs are of great importance when carrying out certain measurements (for instance, reflectance or transmittance evaluation for inhomogeneous objects $[10,11]$ or the assessment of a given source's uniformity) that should otherwise be performed by means of mobile devices. The latter not only are they more time consuming, but they add an additional source of uncertainty to the measurement.

A well-calibrated CCD camera capable of making low-uncertainty measurements would certainly open up new ways in numerous applications since it would offer an increased ability to analyse the recorded information and, as a consequence, it would notably improve the decision-making process.

The simplest way to calibrate, from a radiometric point of view, a CCD detector is to uniformly irradiate it so that each individual detector receives in any given moment in time the same amount of optical radiation. The low-uncertainty calibration of a CCD requires as well the previous design of a stable and uniform radiance source, whose radiance pattern could be measured with low uncertainty.

Moreover, CCD detectors being two-dimensional devices (unlike photodiodes) present additional challenges to the calibration procedure due to charge transfer and other electronic problems. When performing low-uncertainty radiometric measurements each of the CCD-related uncertainty sources must be considered in order to be able to minimize its contribution. In this sense, temporal noise needs to be treated separately from spatial noise, which originates in the variation of the pixel responsivity across the 
array. Charge transfer-related noise also needs to be included, as well as the phenomenon of incomplete collection of the photogenerated electrons. Furthermore, it has to be assessed whether or not the system follows he reciprocity law (between the irradiance and the integration time) and we have to analyze in depth the theoretical linearity between the irradiance and the charge accumulated in the potential wells and between the accumulated charge and the CCD's response (number of counts).

Following this approach, this work presents the experimental setup designed specifically to obtain a lambertian source followed by the procedure devised to callibrate the CCD's responsivity to radiant exposure. The study of the linearity of the CCD's response as well as of the reciprocity between the irradiance and the exposure time have been presented by the authors elsewhere $[13,14]$ and thus, the current work will only focus on their end results.

Finally, the CCD calibration procedure is applied to a particular CCD detector (Sony, ICX414AL). The different sources of uncertainty of the calibration are analyzed and the relative value of each source of uncertainty is calculated based on the expression of the responsivity measurement. It is worth mentioning that the calibration has been performed based on irradiance instead of based on radiance in order to minimize the number of uncertainty sources related to the calibration procedure while highlighting the behavior of the CCD itself and avoiding the uncertainty related to the change of field of view which arises from the introduction of an objective lens in the system when doing radiance measurements.

\section{Experimental setup and calibration proce- dure}

A simplified diagram of the experimental setup designed for the calibration procedure is shown in figure 1. Basically, it consists of a spectral and lambertian irradiance source as well as of a precision positioning system coupled to the CCD and the standard radiometer $* * *$ No me queda muy clara la geometria de la CCD y del radimetro. Uno al lado del otro, cmo les llega la misma irradiancia a los 2 ? No queda claro de la figura. Igual aadira un poco ms de explicacin.....***

that allows to change their location with respect to the sphere's exit port. This setup fulfills all the necessary requirements to provide low-uncertainty measurements [12]. The source consists of an integrating sphere externally illuminated by a $0.02 \%$ powerstabilized dye laser, which, in its turn, is pumped by an Ar laser. This system covers the whole range of wavelengths between $380 \mathrm{~nm}$ and $930 \mathrm{~nm}$, except for the 470-520 nm range, for which a different dye that needs to be pumped by a Kr laser is required.
This range, however, can also be covered by the emission lines of the Ar laser itself. The calibration wavelengths were chosen according to the slope and the curvature of a typical CCD responsivity curve, bearing in mind the potential need for an interpolation. All the wavelengths were obtained using only two dies (550 nm-580 nm, $620 \mathrm{~nm}-700 \mathrm{~nm}$ ) and argon lines (458 nm-514.6 nm). ***Me comentan que el Ar tiene lneas en 457.9 , no en 458 , y en 514.5 , no $514.6 * * *$

The dye laser's bandwidth is equal to $0.05 \mathrm{~nm}$, which means that, for all practical purposes, it can be considered to be a monochromatic source. The laser power entering the sphere is stabilized within $0.02 \%$ by an electro-optical laser power controller.

The integrating sphere has an inner diameter of $50 \mathrm{~cm}$, while for the present calibration it was configured to have an exit port of $12.5 \mathrm{~cm}$ of diameter. We demonstrated elsewhere that this source is uniform and lambertian [15]. A rotating diffuser was placed before the sphere's entrance port in order to minimize the speckle noise of the laser radiation $[17,18]$.

When performing the calibration, it is not recommended to place the CCD exactly at the the exit port's plane in order to avoid radiation from direct incidence and from first reflection. However, placing the CCD elsewhere implies that the irradiance upon each pixel is not constant across the array. The irradiance uniformity was studied as a function of the distance of the CCD to the exit port. For this study the shadow produced by the CCD mount over the CCD itself, the radiation coming from direct incidence and first reflection, the field uniformity [15] and the variation of the responsivity with the solid angle were taken into account. As the pixels shows different responsivity values for different solid angles, it is necessary to know the maximum solid angle that ensures a responsivity variation with respect to normal incidence smaller than the maximum measurement uncertainty that we are aiming for. In order to determine this maximum solid angle, the response of every pixel was evaluated while increasing the distance of the CCD to the exit port. The next step was to determine the threshold distance from which the relative response of the pixels of two photograms recorded for different distance values (both above the threshold one) show a certain degree of correlation. The procedure yielded a threshold distance of $27 \mathrm{~cm}$. From this value the solid angle used for the calibration can be inferred. This angle subtends a maximum incidence angle during the calibration process of 13 .

The absolute responsivity calibration is carried out $[\mathrm{rgb}] 1.00,0.00,0.00^{* * *}$ by direct substitution*** (sustitucin de que en que?), using a 10-ms exposure time. The correct choice of exposure time is vital to the success of the calibration, since a certain nonlinearity factor of the CCD's response can appear for 
interline-type CCDs operating within certain exposure time ranges. For this reason, by means of additional measurements we checked that for exposure time values above $2 \mathrm{~ms}$ the CCD's response is proportional to the radiant exposure [13].

The alignment of the CCD with respect to the sphere's exit port is of great importance. We consider the best alignment to be the one that achieves the most uniform response across the CCD array or, equivalently, the one for which the standard deviation of the pixels's response with respect to the average across the CCD array reaches a minimum value. To find the optimum location of the CCD we employed both the precision positioning system supported by a software routine that calculated every two seconds the abovementioned uniformity value.

As standard radiometer we employed a silicon photodiode of proven linearity with a precision circular aperture of $(8 \pm 0.002) \mathrm{mm}$ of diameter. This photodiode was calibrated against the Spectral Responsivity Scale of the Instituto de Fisica Aplicada (Applied Physics Institute, or IFA) [19], whose standard uncertainty had been determined to be $0.29 \%$.

The equation for the measurement of the radiant exposure responsivity is

$$
R_{i}=\frac{\left(N_{i}-N_{o, i}\right) R_{r a d} F_{i}}{C_{N L} t_{e x p} S_{r a d}}
$$

where $N_{i}$ is the response of the $i$-th pixel, $N_{o, i}$ is the dark signal of the $i$-th pixel, $t_{\exp }$ is the exposure time, $S_{\text {rad }}$ is the radiometer's response, $F_{i}$ represents a factor accounting for the non-uniformity of the field and having a value of 1 in this equation but which needs to be taken into account when estimating the uncertainty of the measurement, $R_{r a d}$ is the irradiance responsivity of the radiometer, and $C_{N L}$ is a correction factor that accounts for the non linearity of the CCD's response. $R_{i}$ represents the corrected responsivity, and has to remain constant for a given wavelength.

It is worth pointing out that $C_{N L}$ should not be mistaken for the previously mentioned nonlinearity. The latter was related to the exposure time, whereas $C_{N L}$ is revealed for measurements having different number of counts, both phenomena having a different origin. The $C_{N L}$ factor can be computed by analyzing how the radiant exposure responsivity changes when varying the number of counts [14]; that is, by calculating the value of $\left(N_{i}-N_{o, i}\right) / t_{\text {exp }}$ for several exposure times within the $[2000 \mu \mathrm{s}-10000 \mu \mathrm{s}]$ interval (this ensures that the non-linearity related to the exposure time does not affect the measurements). This calculation leads to the plot of figure 2, which shows the relation between the relative responsivity and $N_{i}$ (values averaged across all pixels). This function, which describes the relative and normalized evolution of the responsivity, is precisely the correction factor $C_{N L}$. A previous work by these authors [14] previously demonstrated that this function is, at least for the CCD under study, $* * *$ pixel-independent/characterizes all the individual pixels, and not only their average behavior????***.

The calibration procedure was carried out by measuring $R_{i}$ at several wavelengths. Each measurement for a given wavelength was repeated three times to be able to calculate as well the repeatability uncertainty. $N_{i}$ and $S_{\text {rad }}$ are values averaged $* * *$ sobre cuantas repeticiones? ${ }^{* * *}$ in order to minimize temporal noise. The higher the value of $N_{i}$, the lower the CCD's relative noise, which suggest that the count level should be kept as high as possible during the calibration process.

Finally, the calibration procedure thoroughly described in this section was applied to a particular detector, an ICX414AL Sony CCD having 0.5 inches of length and $640 \times 480$ square pixels measuring 9,9 $\mu m$ each. The CCD is integrated in a camera (model Imagen compact) provided with a 12 -bit A/D converter. During the whole calibration process the gain was kept constant to maintain the same responsivity value along the exposure level range.

\section{Results and uncertainty budget}

The result yielded by the CCD's radiometric calibration is the value of the radiant exposure responsivity of each pixel and for each wavelength. This value results from the substitution in equation 1 of the responses registered for each pixel and for the reference standard radiometer. In order to summarize these values, figure 3shows the responsivity averaged across all pixels of the CCD array as a function of the wavelength, where error bars represent the standard deviation of the responsivity. For a homogeneous CCD having few "bad pixels" the standard deviation of the responsivity of the different pixels of the array is relatively low (as can be inferred from the plot) and, therefore, the average value represents an accurate representation of the individual values. For the particular case of the CCD under study, the value of the standard deviation ranged from $0.4 \%$ (at $550 \mathrm{~nm}$ ) to $1 \%$ (at $460 \mathrm{~nm}$ ).

Regarding the value of the responsivity, the curve shows a maximum near $500 \mathrm{~nm}$ and an inflection point at around $600 \mathrm{~nm}$. Since the CCD operates on front illumination and is not equipped neither with a photopic filter nor with any other type of bandpass filter, this particular behavior seems likely to be driven both by the depth of the pixel's space-charge region and by the reflectance of the pixel's surface, which has a very complex structure.

The uncertainty associated to the calculation of 
the responsivity value has been computed by applying the partial derivative rule to equation 1 while assuming that the different variables are uncorrelated. Following this approach, the the total relative uncertainty can be calculated as follows:

$$
u_{r}\left(\Re_{i}\right)=\sqrt{u_{r, C C D}^{2}+u_{r, r a d}^{2}+u_{r, f i e l d}^{2}+u_{r, c a l, i}^{2}}
$$

where $u_{r, C C D}$ represents the CCD-related component of the relative uncertainty, $u_{r, \text { rad }}$ the uncertainty associated to the standard radiometer, $u_{r, \text { field }}$ the uncertainty contribution derived from the nonuniformity of the field, and $u_{r, c a l}$ the uncertainty related to the calibration process. The term $t_{\text {exp }}$ is considered to have no associated uncertainty, and its value is assumed to be the nominal one.

The uncertainty budget is summarized in table 1, where for each uncertainty contribution a representative value is shown (since the value of some of the uncertainty components changes[rgb]1.00,0.00,0.00 $* * *$ with wavelength ${ }^{* * *}$ est bien?).

The total uncertainty never goes over $0.4 \%(k=$ $1)^{* * *}$ qu es $\mathrm{k}$ ? No habra que ponerlo?***. As for the individual contributions, the value of the uncertainty derived from the field's non-uniformity $(0.012 \%)$ has been computed assuming that the sphere's exit port behaves as a uniform and lambertian disk [15]. The standard radiometer's contribution to the total uncertainty $(0.29 \%)$ is obtained from the uncertainty related to the measurement of the detector's area and to that associated to the responsivity scale at the Instituto de Fisica Aplicada (Applied Physics Institute, or IFA), where the authors work. The CCD-related uncertainty accounts for the contributions from the quantization uncertainty, from the charge transfer (which has a greater impact the smaller the amount of transferred charge) [20] as well as from the correction factor $C_{N L}$, which, as mentioned above, is equal to the standard deviation of the fitting performed to obtain the $C_{N L}$-vs.- $N_{i}$ curve [14]. This uncertainty ranges from $0.1 \%$ (high count level) to $0.6 \%$ (low count level). This facts explains why, as was previously mentioned, it is important that the settings are adjusted so that the count level remains relatively high during the calibration process. Finally, the uncertainty associated to the calibration process itself can be derived from the repeatability of the procedure, which is computed from the 3 separate measurements that were registered for each wavelength. This uncertainty, whose value is around $0.15 \%(k=1)$, accounts for alignment errors both of the CCD as well as of the standard radiometer and for other sources of residual temporal noise.

The value of the overall CCD-related uncertainty varies with the number of counts. Figure 4 shows its average value as a function of $N_{i}$, as well as displaying the individual contributions of the various sources of CCD-related uncertainty. As can be derived from the plot, the biggest contributors are the charge transfer process and the non-linearity correction factor. The latter could be minimized, however, if the underlying physical process responsible for the lack of linearity was better understood. The quantization noise is only significant for low count levels, which indicates that increasing the number of bits of the $\mathrm{A} / \mathrm{D}$ converter doesn't really help to decrease the associated uncertainty. $* * *$ no entiendo que pinta esta frase aqui $* * *$

The total uncertainty of the radiant exposure responsivity of the CCD together with its individual contributions are shown in figure 5 , in this case as a function of wavelength. The variation of the uncertainty value with wavelength is due to two reasons. First, the repeatability is not the same for each wavelength; second, the count level attained during the calibration also varied, since for some wavelengths only low power levels were available. This fact, as mentioned before, has an important impact upon the CCD-related uncertainty value.

To summarize, the resulting uncertainty contribution excluding the one related to the standard radiometer is about $0.18 \%(\mathrm{k}=1)$, so the calibration procedure can be considered good. *** por qu 0,18 es bueno? ***

This calibration procedure doesn't cover the dependency of the process with temperature or with the value of the solid angle of illumination. For the calibration described in this work the operating temperature was kept at $22 \mathrm{C}$ and the field of view was restricted to values between 0 and 13 .

\section{Conclusions}

We have developed a low-uncertainty calibration procedure of the radiant exposure responsivity of CCDs. The results show that under constant temperature conditions and if the field of view is kept under $0.13 \%$, the uncertainty associated to the calibration method is approximately equal to $0.18 \%$.

The different components of the calibrationrelated uncertainty have been analyzed separately, which allowed us to find out that those associated to the repeatability of the procedure and to the CCD itself account for the highest relative impact upon the total uncertainty value. Among the CCD-related uncertainty components, those derived from charge transfer noise and from the non-linearity correction are the most important ones. On the other hand, quantization noise is only significant for low values of the number of counts, which means that increasing the number of bits of the A/D converter ***doesn't decrease the uncertainty value/is not really important for the current procedure***. 
The overall uncertainty of the responsivity under the conditions described in the present paper is very close to that of the spectral responsivity scale upon which this calibration is based, which demonstrate the goodness of the method. [rgb]1.00,0.00,0.00La incertidumbre final de la responsividad en las condiciones medidas es muy prxima a la de la escala de responsividad espectral en la que se basa esta calibracin, lo que pone de manifiesto la bondad del mtodo. ${ }^{* * *}$ Lo he traducido pero No entiendo este parrafo $* * *$

\section{Acknowledgements}

This work has been partially supported by the thematic network DPI2002-11636-E and by project DPI2001-1174-C0201.

Table 1. Uncertainty budget for the calibration of the CCD.

\begin{tabular}{cc} 
Uncertainty source & $\begin{array}{c}\text { Typical value } \\
(\%)(\mathbf{k}=\mathbf{1})\end{array}$ \\
\hline CCD & 0.1 \\
Charge transfer & 0.01 \\
Quantization & 0.1 \\
Nonlinearity factor & \\
Radiometer responsivity & 0.29 \\
Flux responsivity & 0.025 \\
Area & 0.012 \\
Field uncertainty & 0.15 \\
Repeatibility & $\mathbf{0 . 3 5}$ \\
\hline Total &
\end{tabular}

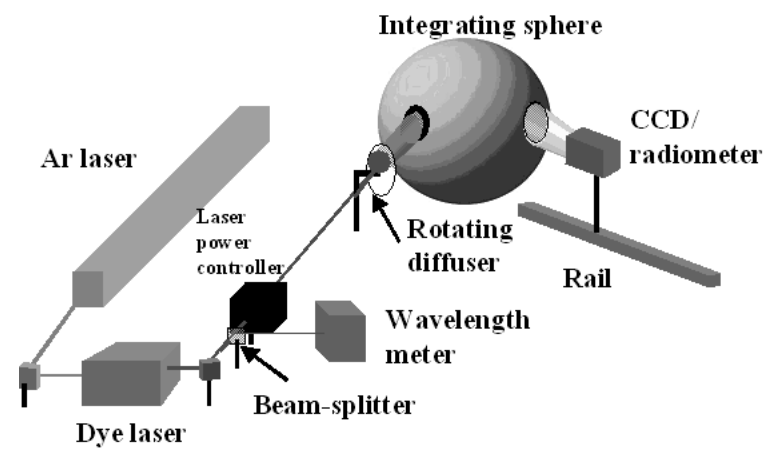

Figure 1. Radiance source for the radiometric calibration.
Table 2. Espectral radiant exposure responsivities values and associated expanded uncertainties

\begin{tabular}{ccc}
$\begin{array}{c}\text { Wavelength } \\
(\mathbf{n m})\end{array}$ & $\begin{array}{c}\text { Responsivity } \\
\left(\text { counts } \boldsymbol{\mu} \mathbf{s}^{-1} \mathbf{W}^{-1} \mathbf{m}^{2}\right)\end{array}$ & $\begin{array}{c}\text { Expanded } \\
\text { uncertainty }(\%)\end{array}$ \\
\hline 458 & 10.70 & 0.72 \\
476.6 & 12.76 & 0.67 \\
488.1 & 13.93 & 0.70 \\
496.6 & 14.42 & 0.70 \\
501.8 & 14.60 & 0.66 \\
514.6 & 14.38 & 0.64 \\
550 & 12.56 & 0.69 \\
560 & 12.09 & 0.77 \\
570 & 11.77 & 0.67 \\
580 & 11.61 & 0.74 \\
620 & 10.87 & 0.78 \\
640 & 10.47 & 0.68 \\
660 & 9.73 & 0.67 \\
680 & 8.75 & 0.67 \\
700 & 7.64 & 0.81 \\
\hline
\end{tabular}

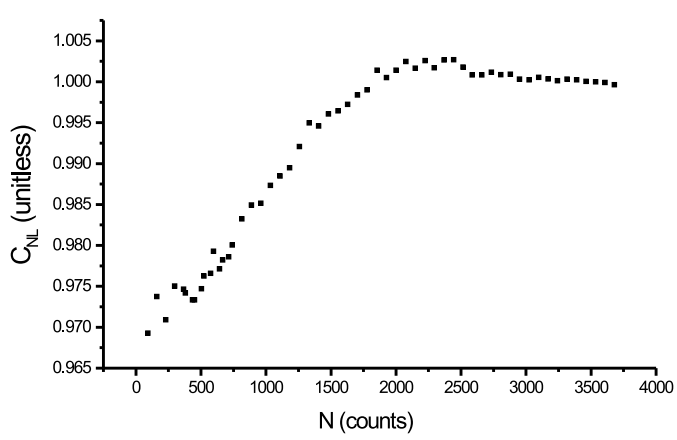

Figure 2. Valores experimentales del factor de correccin de la no linealidad.

\section{References}

1. W. Boyle and G. Smith, "Charge coupled devices," Bell Syst. Tech. J., 49, pp. 587-593 (1970).

2. J. R. Janesick, "History, Operation, Performance, Design, Fabrication and Theory," en Scientific ChargeCoupled Devices, ed. (SPIE Press, Bellingham, Washington, USA, 2001), pp. 3-94.

3. R. Gentile, P. Allebach and E. Wallowitt, "Quantization of Colour Images Based on Uniform Color Spaces," J. of Imag. Techn., 16, pp. 11-21 (1990).

4. P. Iacomussi and M. Rastello, "Colorimetry of 3 CCD Video Cameras," CIE Publication 133, Vienna, International Commission on Illumination, p. 65 (1999).

5. M. Simpson and J. Jansen, "Imaging Colorimetry: a new approach," Applied Optics, 30, 4666-4671 (1991).

6. G. Rossi, P. Iacomussi and P. Soardo, "A CCD multidetector for the measurement of the luminous in- 


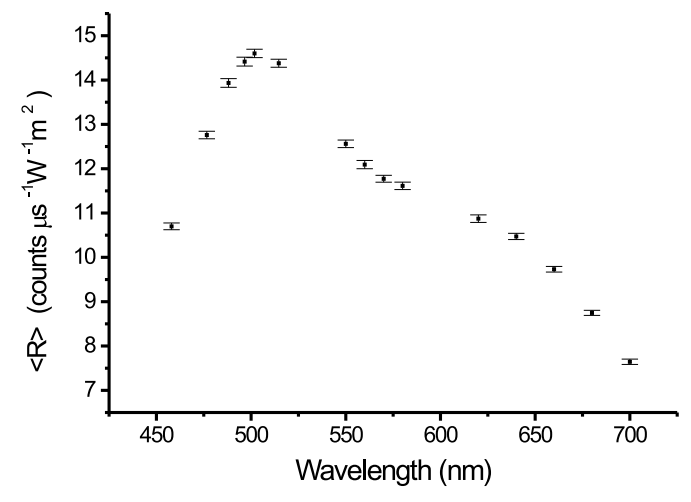

Figure 3. Average spectral responsivity of a CCD Sony ICX414AL.

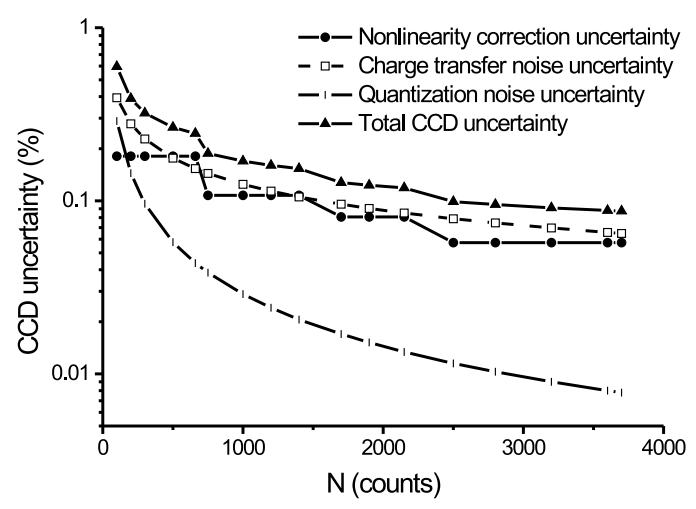

Figure 4. CCD uncertainty components.

tensity distribution of a luminaire at short distance," CIE Publication 119, Vienna, International Commission on Illumination, pp. 130-131 (1995).

7. L. Bellia, A. Cesarano, F. Minichiello and S. Sibilio, "A CCD photometer for lighting research and design," CIE Publication 133, Vienna, International Commission on Illumination, pp. 219-221 (1999).

8. P. Longre and A. Trmeau, "Color Appearance: Effects of Texture and Relief," SPIE Proc., 3409, 89-97 (1998).

9. G. Healey and R. Kondepudy, "Radiometric CCD Camera Calibration and Noise Estimation," IEEE Trans. Patt. Anal. Mach. Int., 16, 267-276 (1994).

10. J. Dymond and M. Trotter, "Directional reflectance of vegetation measured by a calibrated digital camera," Applied Optics, 36, 4314-4319 (1997).

11. S. Nishida and M. Shinya, "Use of image-based information in judgements of surface-reflectance properties," J. Opt. Soc. Am. A, 15, 2951-2965 (1998).

12. J. Campos, "Radiometric calibration of chargecoupled-device video cameras," Metrologia, 37, 459464 (2000).

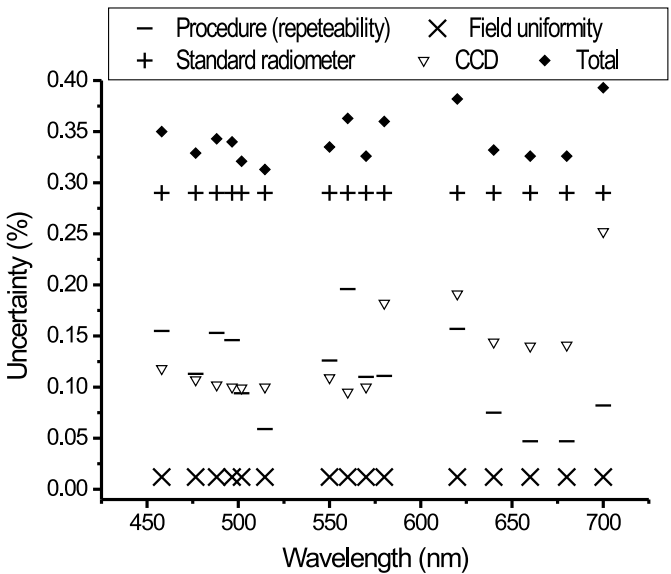

Figure 5. Uncertainty budget of the radiant exposure responsivity calibration as a function of wavelength.

13. A. Ferrero, J. Campos and A. Pons, "Apparent violation of the radiant exposure reciprocity law in interline CCDs," (sent to Applied Optics), 2005.

14. A. Ferrero, J. Campos and A. Pons, "Non-uniformity correction procedure for matrix detectors based on the prior compensation of its nonlinear behavior," (sent to Applied Optics), 2005.

15. A. Ferrero, J. Campos and A. Pons, "Radiance source for CCD absolute radiometric calibration", NewRad, Davos, 2005.

16. International Lighting Vocabulary, Publication CIE $17.4,1987$.

17. S. Lowenthal and D. Joyeux, "Speckle Removal by a Slowly Moving Diffuser Associated with a Motionless Difusser," J. Opt. Soc. Am., 61, No. 7, 847-851 (1971).

18. E. Schröder, "Elimination of granulation in laser beam projections by means of moving diffusers," Optics Communications, 3, No. 1, 68-72 (1970).

19. J. Campos, A. Pons and P. Corredera, "Spectral Responsivity Scale in the Visible Range based on single silicon photodiodes," Metrologia, 40, S181-S184 (2003).

20. J. R. Janesick, "Transfer noise," in Scientific ChargeCoupled Devices, (SPIE Press, Bellingham, Washington, USA, 2001), p. 656.

Received on xx xxxx 2005. 